\title{
SÍNDROME DE FRAGILIDAD EN EL ADULTO MAYOR EN UNA COMUNIDAD RURAL DE PUEBLA, MÉXICO
}

\section{SYNDROME OF FRAILTY IN THE OLDER ADULT IN A RURAL COMMUNITY IN PUEBLA, MEXICO}

\section{TÍTULO CORTO: SÍNDROME DE FRAGILIDAD EN EL ADULTO MAYOR}

\author{
Liliana Rivadeneyra-Espinoza ${ }^{1}$, Cristina del Rocio Sánchez-Hernández ${ }^{2}$
}

Recibido en octubre 02 de 2015

Aceptado en marzo 16 de 2016

\section{RESUMEN}

El síndrome de fragilidad en el adulto mayor implica presentar eventos adversos, mayor necesidad de cuidados, mayor riesgo de evolucionar hacia la discapacidad, dependencia y muerte. El objetivo de la investigación fue determinar el número de adultos mayores que presentan síndrome de fragilidad y el factor más determinante, en una población rural del estado de Puebla, México. Se realizó un estudio descriptivo, observacional, transversal, prospectivo. La muestra estuvo conformada por 200 pacientes mayores de 65 años de edad, registrados en el centro de salud de la comunidad en estudio. Los datos fueron recolectados a través de la aplicación de la escala geriátrica y se aplicaron los criterios cubanos de fragilidad. De los adultos mayores incluidos $52.5 \%$ fueron femeninos y 47.5 masculinos. La prevalencia del síndrome de fragilidad fue del $45 \%$, siendo mayor en mujeres. Como factor asociado se encontraron las alteraciones en la movilidad y el equilibrio con un $26.5 \%$. Se concluye que existe una alta prevalencia de adultos mayores frágiles en una comunidad rural de Puebla, México; las alteraciones en la movilidad y el equilibrio son el principal factor detonante, debido a las actividades cotidianas que predominan en las zonas rurales latinoamericanas.

Palabras clave: Adulto mayor; Fragilidad; Geriatría

\section{ABSTRACT}

Syndrome of frailty in the elderly, involves presenting adverse events, most in need of care, increased risk of progression to disability, dependency and death. The objective of the research was to determine the number of older adults presenting fragility syndrome and the most determining factor in a rural population of the State of Puebla, Mexico. A descriptive, observational, cross-sectional, prospective study was conducted. The sample was composed of 200 patients over 65 years of age, registered in the study community health center. Data was collected through the application of the geriatric scale and fragility of Cuban criteria were applied. Including older adults $52.5 \%$ were female and 47.5 male. The prevalence of fragility Syndrome was $45 \%$, being higher in women. As associated factor

1. Maestría en Ciencias. Catedrático investigador, Universidad Popular Autónoma del Estado de Puebla, México. Correo: liliana.rivadeneyra@upaep.mx

2. Médico General. Pasante del Servicio Social en el área de investigación, Universidad Popular Autónoma del Estado de Puebla, México. Correo: dra.cristina.sanchez@ hotmail.com 
found alterations in mobility and balance with a $26.5 \%$. It is concluded that there is a high prevalence of fragile seniors in a rural community in Puebla, Mexico; alterations in mobility and balance are the main detonating factor, due to activities of daily living that predominate in Latin American rural areas.

Keywords: Older adult; Frailty; Geriatric

\section{INTRODUCCIÓN}

$E^{1}$ aumento de la población adulta mayor constituye hoy un elemento primordial en investigaciones médicas y sociales, y aunque es importante la longevidad también lo es que esta transcurra con una mejor calidad de vida para el adulto mayor ${ }^{1}$. El porcentaje de población de adultos mayores de 60 años o más en el estado de Puebla, México en el 2010 fue de $9.0 \%$ aumentando casi $2 \%$ con respecto al año 2005 que fue de $8.40 \%{ }^{2}$. Por esta razón, conocer las condiciones que colocan al adulto mayor en situaciones de riesgo, son pauta de nuevas investigaciones del área de la salud, así como de las ciencias sociales, económicas, entre otras; que se han dirigido al proceso fisiológico de envejecimiento, en el cual hay pérdida de la reserva funcional, condicionando a una mayor susceptibilidad, disminución de los mecanismos de respuesta y la eficacia para conservar la homeostasis ${ }^{3-5}$. El término de fragilidad ha surgido en un intento de poder diagnosticar a un adulto mayor que requiere apoyo de varios servicios para manejarse en las actividades de la vida diaria.

El paciente frágil es aquel que se encuentra delicado de salud, débil y en un estado de vulnerabilidad. El síndrome de fragilidad en el adulto mayor implica una situación de presentar riesgos adversos (deterioro funcional, mortalidad, hospitalización e institucionalización), mayor necesidad de cuidados, mayor riesgo de evolucionar hacia la discapacidad, dependencia y muerte ${ }^{6,7}$; además, los adultos mayores frágiles generan gran impacto sobre el sistema de salud debido a que son mayores consumidores de medicamentos y servicios hospitalarios, representando un aumento en los gastos de salud y asistencia social. El diagnóstico de forma temprana de este síndrome en este sector de la población modifica de forma esperada su calidad de vida, implementando programas preventivos con el propósito de evitar el progreso del síndrome en estas personas, por ejemplo: evitar las consecuencias de la polifarmacia o establecer rutinas de ejercicios que le permitan mejorar su movilidad.
En la base de estudios de Estados Unidos se mostró que el $7 \%$ de los adultos mayores de 65 años son frágiles, aumentando progresivamente con la edad hasta el 25$40 \%$ en mayores de 80 años ${ }^{8}$. Otro estudio realizado en el año 2009 en una comunidad urbana de Cuba, evidenció que el $51.4 \%$ de los estudiados eran frágiles, siendo la polifarmacia el factor de prevalente en un $35.5 \%$ y el sexo femenino tiene un riesgo relativo de ser más frágil con respecto al sexo masculino ${ }^{1}$. Varios estudios se han realizado acerca de este síndrome en los cuales no se observan diferencias en las variables que resultan asociadas al síndrome de fragilidad: edad avanzada, sexo femenino, bajo nivel educativo, pocos ingresos económicos, comorbilidad, polifarmacia y discapacidad ${ }^{6-8}$.

A lo largo del tiempo se han utilizado diversos criterios e instrumentos para el diagnóstico de fragilidad en el adulto mayor, tal como es el cuestionario de Barber, sin embargo algunos reportes cuestionan su utilidad ya que ha sido integrado con base a criterios de expertos, más que a evidencias científicas ${ }^{5}$; otros métodos empleados para el diagnóstico de este síndrome son los criterios cubanos de fragilidad, los cuales se derivan de la aplicación de la escala geriátrica de evaluación funcional de la cual se obtiene el estado funcional global del adulto mayor, calculado por el promedio de las doce variables (EGEF) ${ }^{1}$.

Los criterios Fried validan un fenotipo de fragilidad en el adulto mayor con base en 5 aspectos clínicos: pérdida de peso no intencionada, debilidad o agotamiento, lentitud en la movilidad, debilidad muscular, y una baja en el nivel de actividad física, uno de los principales obstáculos para su correcta aplicación es la poca practicidad para para detectar a los ancianos frágiles a nivel comunitario, ya que se necesitan obtener valoraciones complejas que requieren mucho tiempo y necesitan su transcripción ${ }^{6,9,10}$. Se han aplicado tests funcionales de observación directa destinados a cuantificar la limitación funcional, los cuales han podido identificar a ancianos vulnerables en riesgo de presentar episodios adversos, de estos destaca el 
Short Physical Performance Battery, que se relaciona con discapacidad incidente y con mortalidad. Otro método empleado para la detección del adulto mayor frágil es el índice de fragilidad denominado Rockwood como se cita en Abizanda et $\mathrm{al}^{11}$ el cual se basa en cuatro grados de discapacidad progresiva, incluye actividades de la vida diaria básica (ABVD), de ambulación, continencias y cognición. La escala de impresión cllínica global de cambio en fragilidad física, Studenski como se reporta en Abizanda et $\mathrm{al}^{12}$ incluye movilidad, equilibrio, fuerza, resistencia, nutrición y función neuromotora.

Hasta la fecha no existe consenso sobre los instrumentos que se deben aplicar por el médico para detectar este síndrome por lo tanto el síndrome de fragilidad sigue siendo un terreno confuso. Básicamente los criterios de fragilidad han sido agrupados en cuatro categorías: médicos, funcionales, mentales y sociodemográficos ${ }^{13}$. En lo que sí parece haber un consenso, es en el hecho de que al realizar una valoración geriátrica integral sistemática en los adultos mayores puede reconocerse el síndrome de fragilidad en el nivel primario de atención medica; tales de los criterios cubanos que parten de la escala geriátrica de la evaluación funcional del adulto mayor. Este nivel de atención médica primaria es el encargado de iniciar estrategias para promover la salud y la educación en este sector de la población.

El objetivo de la investigación fue determinar el número de adultos mayores que presentan el síndrome de fragilidad y el factor más determinante, en una población rural del estado de Puebla, México, en el otoño del 2014.

\section{MATERIALES Y MÉTODOS}

En esta investigación se utilizó un diseño cuantitativo, descriptivo y observacional, transversal prospectivo.

La selección de la muestra no fue de manera probabilística ya que se basó en criterios de inclusión, exclusión y eliminación. Se consideraron a 200 pacientes los cuales deberían poseer residencia oficial en el pueblo de San Matias Tlalancaleca en el estado de Puebla, tener más de 65 años de edad, estar registrados en el centro de salud de dicho municipio, expresar conformidad de participar en dicho estudio o ser cuidador crucial de un adulto mayor en caso de que él no pueda contestar el cuestionario por las condiciones de salud. Se excluyeron los adultos mayores que no tenga residencia de más de 5 años en dicho municipio o que no deseara participar en la investigación. Los cuestionarios incompletos o de adultos mayores que presenten problemas auditivos al momento de realizarle el cuestionario y que no contaba con un cuidador fueron eliminados.

Se aplicó la escala geriátrica de evaluación funcional para obtener los criterios cubanos de fragilidad que presente el adulto mayor estudiado y así clasificarlo en ancianos frágiles y no frágiles. Esta escala tipo Likert consta de doce variables: continencia, movilidad, equilibrio, visión, uso de medicamentos, sueño, estado emocional, memoria y situación económica. A las cuales se les asignan valores del 1-5 en dependencia de la situación específica de salud del adulto mayor evaluado, posteriormente se obtiene una variable en base al promedio de las 12 variables y se consigue el estado funcional global del adulto mayor. Al final en base a los criterios cubanos de fragilidad se clasifica en adulto mayor frágil si presenta un criterio o adulto mayor no frágil, si no presenta ningún criterio ${ }^{1}$.

\section{Declaración de aspectos éticos}

En lo referente a los aspectos éticos, esta investigación se basó en la 4 Ley General de Salud en materia de Investigación en México, la cual contempla en su artículo $14^{\circ}$ que toda aquella investigación que se realice en seres humanos deberá contar con el consentimiento informado del sujeto de investigación o su representante legal. Por lo que para poder realizar la encuesta se contó con la autorización de la persona.

\section{RESULTADOS}

Se estudiaron 200 adultos mayores residentes de una población rural del estado de Puebla, de los cuales el $52.5 \%$ pertenecían al sexo femenino y el $47.5 \%$ al masculino; la edad promedio de la población total fue de 74.8, en el sexo femenino de 74.0 y de 75.7 en el sexo masculino, con edad mínima de 65 años y máxima de 102 años.

A todos los adultos mayores se les aplicó la escala geriátrica de evaluación funcional (EGEF). Según los resultados de esta evaluación se estima que en la mayoría de las variables los adultos mayores están clasificados predominantemente en las categorías 5 y 4 que corresponde a la puntuación de cada variable, es decir, no son limitantes en sus actividades en la vida diaria.

En cuanto a la "movilidad" se identifica que el 32.5\%, es decir, 3 de cada 10 personas presentan alteraciones en la movilidad predominando en el sexo femenino. En 
la variable "equilibrio", 2 de cada 10 adultos mayores presentan trastornos del equilibrio repercutiendo en sus actividades de la vida diaria. El $23.5 \%$ de estos presentan alteraciones visuales que limitan sus actividades cotidianas; es decir, 2 de cada 10 adultos mayores. En el caso de "uso de medicamentos" el $21.5 \%$ de la población consume medicamentos, de estos 32 consumen entre tres a cinco medicamentos, y el resto más de 6 medicamentos, el uso de fármacos se presenta más en el sexo femenino. Se evidenció una frecuencia de depresión $11 \%$ (22 personas) de las cuales la mayoría (18) usa psicofármacos. El 14\% de los adultos mayores padece trastornos de la memoria con riesgo a ser dependientes parte del tiempo. (Tabla 1)

Tabla 1. Distribución de los adultos mayores de acuerdo al resultado de la EGEF aplicada en una población rural del estado de Puebla.

\begin{tabular}{|c|c|c|c|c|c|c|c|c|c|c|}
\hline \multirow{2}{*}{ VARIABLE } & \multicolumn{9}{|c|}{ PUNTUACION DE LA VARIABLE } \\
\cline { 2 - 15 } & \multicolumn{2}{|c}{5} & \multicolumn{2}{|c|}{4} & \multicolumn{2}{c|}{3} & \multicolumn{2}{c|}{2} & \multicolumn{2}{c|}{1} \\
\cline { 2 - 16 } & TOTAL & $\%$ & TOTAL & $\%$ & TOTAL & $\%$ & TOTAL & $\%$ & TOTAL & $\%$ \\
\hline 1.-Continencia & 104 & 52 & 54 & 27 & 29 & 14.5 & 9 & 4.5 & 4 & 2 \\
\hline 2.-Movilidad & 79 & 39.5 & 56 & 28 & 47 & 23.5 & 14 & 7 & 4 & 2 \\
\hline 3.-Equilibrio & 72 & 36 & 76 & 38 & 38 & 19 & 12 & 6 & 2 & 1 \\
\hline 4.-Vision & 47 & 23.5 & 106 & 53 & 31 & 15.5 & 12 & 6 & 4 & 2 \\
\hline 5.-Audicion & 83 & 41.5 & 75 & 37.5 & 28 & 14 & 14 & 7 & 0 & 0 \\
\hline 6.-Uso De Medicamentos & 68 & 34 & 89 & 44.5 & 32 & 16 & 8 & 4 & 3 & 1.5 \\
\hline 7.-Sueño & 106 & 53 & 71 & 35.5 & 19 & 9.5 & 3 & 1.5 & 0 & 0 \\
\hline 8.-Estado Emocional & 84 & 42 & 94 & 47 & 18 & 9 & 2 & 1 & 2 & 1 \\
\hline 9.-Memoria & 53 & 26.5 & 119 & 59.5 & 20 & 10 & 8 & 4 & 0 & 0 \\
\hline 10.-Apoyo Familiar & 42 & 21 & 106 & 53 & 33 & 16.5 & 18 & 9 & 1 & 0.5 \\
\hline 11.-Apoyo Social & 38 & 19 & 85 & 42.5 & 56 & 28 & 18 & 9 & 4 & 2 \\
\hline 12.-Situacion Económica & 32 & 16 & 132 & 66 & 29 & 14.5 & 8 & 4 & 0 & 0 \\
\hline 13.-Edo Funcional Global & 39 & 19.5 & 116 & 58 & 40 & 20 & 5 & 2.5 & 0 & 0 \\
\hline
\end{tabular}

Respecto al "apoyo social” el 39\% refiere una relación limitada con los vecinos en situaciones de crisis, así mismo, más de la mitad de los adultos mayores (82\%) refiere que sus ingresos cubren sus necesidades básicas (Tabla 1).

122 La situación funcional global calculada por las doce variables de la EGEF, muestra que el $77.5 \%$ de la población adulta mayor es independiente, el $22.5 \%$ de la población es dependiente; y de estos la mayoría tienen limitaciones que exigen ayuda diaria, se identificó que el sexo femenino tiene una situación funcional global mejor con respecto al sexo masculino (Tabla 1).

En base a los criterios cubanos de fragilidad 90 adultos mayores de esta comunidad se clasificaron con síndrome de fragilidad; el $27 \%$ de la población femenina (54) y el 18\% (36) de la población masculina es frágil (Tabla 2). 
Tabla 2. Distribución de los adultos mayores de acuerdo al estado de fragilidad y género.

\begin{tabular}{|c|c|c|c|c|c|c|}
\hline \multirow{2}{*}{ SEXO } & \multicolumn{2}{|c|}{ ADULTO MAYOR FRÁGIL } & \multicolumn{2}{c|}{$\begin{array}{c}\text { ADULTO MAYOR NO } \\
\text { FRÁGIL }\end{array}$} \\
\cline { 2 - 7 } & TOTAL & $\%$ & TOTAL & $\%$ & TOTAL & $\%$ \\
\hline FEMENINO & 54 & 27 & 51 & 25.5 & 105 & 52.5 \\
\hline MASCULINO & 36 & 18 & 59 & 29.5 & 95 & 47.5 \\
\hline TOTAL & 90 & 45 & 110 & 55 & 200 & 100 \\
\hline
\end{tabular}

De acuerdo al 95\% del nivel de confianza en el tamaño de la población total de 1000 adultos mayores se estimó un tamaño representativo de la muestra de 200 individuos, los cuales 90 presentaron síndrome de fragilidad, se aplicó la fórmula de prevalencia (número de casos existentes o enfermos entre el número total de individuos en la población) teniendo como resultado el $45 \%$ en esta comunidad rural.

Al evaluar la relación de fragilidad con la edad se aprecia una tendencia de presentar el síndrome conforme avanza la edad. En las edades comprendidas de 60 a 69 años, los adultos mayores en su mayoría no son frágiles, en las edades comprendidas entre los 70 a 79 años aumenta el porcentaje de adultos mayores frágiles, llegando al
$100 \%$ en los adultos mayores de 90 años. Es importante destacar que la fragilidad aparece más tempranamente en el género femenino.

En orden de prevalencia el criterio de fragilidad "alteraciones de la movilidad y equilibrio" se presenta en el $26.5 \%$ (53) de los adultos mayores, el $29.5 \%$ pertenece al género femenino (105) y el $23.1 \%$ (95) al género masculino. Este criterio va seguido de "cualquier combinación de los problemas sociales" con un $21.5 \%$, con predominio en la población femenina. En tercer lugar, se encuentra la "polifarmacia" con un $16.5 \%$, de este el género femenino representa un $20 \%$. La frecuencia con que se presentan los criterios cubanos de fragilidad y el porcentaje que representan con respecto a la población general se visualiza en la Tabla 3.

Tabla 3. Distribución de los adultos mayores en base a los criterios cubanos de fragilidad y género.

\begin{tabular}{|l|c|c|c|}
\hline \multicolumn{1}{|c|}{ CRITERIOS CUBANOS DE FRAGILIDAD } & FEMENINO & MASCULINO & TOTAL \\
\cline { 2 - 4 } & $\% \mathrm{~N}=105$ & $\% \mathrm{~N}=95$ & $\% \mathrm{~N}=200$ \\
\hline 1.-Doble incontinencia & 1.9 & 3.1 & 2.5 \\
\hline 2.-Alteraciones de la movilidad y el equilibrio (< 4 según EGEF). & 29.5 & 23.1 & 26.5 \\
\hline 3.-Polifarmacia (uso de 3 o más medicamentos) & 20 & 12.6 & 16.5 \\
\hline 4.-Alteraciones de todas las variables de la EGEF en 4 o menos. & 15.2 & 12.6 & 14 \\
\hline 5.-Antecedentes patológicos personales de síndrome demencial & 0.9 & 1 & 1 \\
\hline 6.-Cualquier combinación de los problemas sociales (<4 según EGEF). & 25.7 & 16.8 & 21.5 \\
\hline 7.-Anciano con alguna alteración del EGEF. & 13.3 & 8.4 & 11 \\
\hline 8.-Mayor de 80 años con alguna alteración del EGEF. & 16.1 & 22.1 & 16 \\
\hline 9.-Alteraciones de la memoria (< 4 según EGEF). & 10.4 & 12.6 & 11.5 \\
\hline 10.-Alteraciones del estado funcional global (<4 según EGEF). & 16.1 & 22.1 & 19 \\
\hline
\end{tabular}


Explorando el número de criterios por adulto mayor frágil se identificó que el $73.5 \%$ tiene de 1 a 4 criterios (categoría 1), el $25.5 \%$ presenta de 5 a 7 criterios (categoría 2) y solo el $1.1 \%$ tiene de 8 a 10 criterios (categoría 3).

Dentro de las categorías antes mencionadas en la 1 el criterio prevalente es "alteraciones de la movilidad y equilibrio", en la categoría 2 fue "problemas sociales"; estos dos criterios se presentaron con mayor frecuencia en el género femenino.

\section{DISCUSIÓN}

Una oportuna detección y asesoramiento en el tratamiento del del adulto mayor frágil resulta un punto clave dentro de la practica en la medicina geriátrica ${ }^{14}$. El tema de fragilidad sigue siendo controversial, principalmente porque no existe un consenso universal sobre cuales criterios se deben utilizar para su diagnóstico. En lo que si hay un acuerdo es que la fragilidad representa en el adulto mayor una alta susceptibilidad de cambios fisiológicos a consecuencia de la deteriorada capacidad funcional que poseen, para enfrentar diversas situaciones de estrés ${ }^{14}$, como lo utilizado en los criterios cubanos. Se han descrito estudios donde el síndrome de fragilidad oscila entre el 6 y $98 \%$, tomando en cuenta a un adulto mayor de $65 \mathrm{annos}^{15-18}$. En el estudio piloto "criterios de fragilidad del adulto mayor" realizado en la ciudad de Lleida España, utilizaron los criterios de Fried modificados por demostraron la presencia del síndrome de fragilidad de un $8.5 \%$ y como factores asociados a la fragilidad fueron el sexo femenino y pocos ingresos mensuales $^{6}$. Otro estudio del síndrome de fragilidad realizado en la habana cuba "Diagnostico de fragilidad en adultos mayores de una comunidad urbana" identifico como frágil al 54\% de los estudiados predominando en el género femenino, siendo la polifarmacia fue el criterio con mayor asociación, en este estudio el porcentaje de fragilidad resulto menor y a diferencia de ellos la polifarmacia no fue el criterio con mayor asociación.

Se estimó la prevalencia de este síndrome siendo de un $45 \%$, aplicando los criterios cubanos de fragilidad y la escala geriátrica de evaluación funcional. En este estudio los factores mayormente asociados fueron las alteraciones en la movilidad y equilibrio, cualquier combinación de los problemas sociales y polifarmacia. El más frecuente de ellos "alteraciones en la movilidad y equilibrio", es una de las condiciones que se asocia con fragilidad en el adulto mayor, a diferencia de otros estudios, este criterio llega a ocupar el tercer lugar y la polifarmacia el primero ${ }^{1,10,12}$, en este estudio a diferencia de lo mencionado las alteraciones de la movilidad y equilibrio están muy por arriba de la polifarmacia, $26.5 \%$ y $16.5 \%$ respectivamente, el aumento de la preponderancia de esta alteración en la población, representa una alerta sobre los cambios en la condición de riesgo del adulto mayor. Es muy diferente la situación de riesgo que pudiera tener un adulto mayor sin alteraciones en la movilidad y equilibrio a uno que la presente, considerando que estos individuos con este criterio tienen limitaciones en satisfacer sus actividades de la vida diaria, es dependiente de otra persona, lo predisponen a caídas y a la discapacidad ${ }^{19}$. En esta población el alto predomino de estas alteraciones pueden estar asociadas al estilo de vida de los adultos mayores, a causa de que la mayoría de ellos se dedican a las actividades agrícolas, ganaderas y de carga, además la infraestructura social logra influir en el estado de salud de los adultos mayores frágiles pues existen aún calles sin pavimento, limitando la movilidad del adulto mayor y predisponiéndolo a caídas, esta situación es compartida en muchas poblaciones rurales de México. Los problemas sociales también están asociados al síndrome de fragilidad ${ }^{1,6}$, como una consecuencia del tener pocos ingresos económicos los cuales solo llegan a cubrir sus necesidades básicas y el apoyo familiar restringido a situaciones de crisis, lleva al adulto mayor a un contexto de asistencia social y sanitaria deficiente, implicando un deterioro más acelerado de la salud del adulto mayor y por lo tanto mayor tendencia a padecer eventos agudos de enfermedades crónicas que padezca, disminución de la capacidad funcional, aumentando así el consumo de medicamentos ${ }^{20}$. Esta falta de apoyo familiar se encuentra marcada en este tipo de poblaciones rurales como en muchos países de Latinoamérica a consecuencia de la migración de los hijos hacia zonas urbanas. Por otra parte, la condición de polifarmacia se asocia con mayor frecuencia en el género femenino al igual que en otros estudios realizados ${ }^{1}$.

Finalmente con respecto a la relación entre género y fragilidad, los resultados de esta investigación coinciden con otros estudios en los cuales se plantea que existe asociación entre el género femenino y el síndrome de fragilidad en el adulto mayor ${ }^{1,6,18,19}$, este hallazgo común se puede explicar por lo presentado por Jurschik et $\mathrm{al}^{5}$ donde refieren que este hallazgo está explicado por la alta predisposición del género femenino a sufrir sarcopenia, sustentando así fisiológicamente la fragilidad por deterioro del estado físico a causa de la pérdida de masa muscular.

Al analizar la bibliografía disponible y los resultados de esta investigación, se hace evidente que la incidencia de fragilidad y de cada uno de los criterios, está determinada por los contextos diferentes en los cuales se desarrolla el adulto mayor, como la actividad de campo importante que predomina aun en las zonas rurales latinoamericanas. 
La población rural en estudio del estado de Puebla cuenta con una población de 1000 adultos mayores de más de 65 años y se estima que para el año 2020 la población sea a 1068, por ello deben crearse programas priorizados a la atención integral del adulto mayor, el cual debe contemplar el diagnostico de síndrome de fragilidad en la evaluación integral del adulto mayor, mediante una asistencia de calidad de todos los servicios de salud, debido a que cobra una gran relevancia el permitir la prevención y la intervención, modificando así la expresión de fragilidad en un adulto mayor.

\section{DECLARACIÓN SOBRE CONFLICTO DE INTERÉS}

Se deja constancia que los autores no tienen conflictos de interés y que este trabajo no ha tenido fuentes de financiamiento en ninguna de sus etapas.

\section{REFERENCIAS BIBLIOGRÁFICAS}

1. Alonso P, Sanso F, Díaz A, Carrasco M. Diagnóstico de fragilidad en adultos mayores de una comunidad urbana. Revista Cubana de Salud Pública. 2009; 35(2): 1-14.

2. Instituto Nacional de Estadística y Geografía (INEGI). [citado 13 mayo 2015].Disponible en: http://www3. inegi.org. $\mathrm{mx} /$ sistemas $/$ mexicocifras/default.aspx? $\mathrm{e}=21$

3. Romero R. El instrumento de fragilidad para atención primaria de la encuesta de salud, envejecimiento y jubilación en Europa (SHARE-FI): resultados de la muestra española. Revista Española de Geriatría y Gerontología. 2011; 46(5):243-249.

4. Buchner D, Wagner E. Preventing frail health. Revista Clin Geriatr Med.1992;8:1 17

5. Jurschik P, Escobar M, Nuin C, Botigue T. Criterios de fragilidad del adulto mayor. Estudio piloto. Revista Elsevier. 2011; 43(4):190-196.

6. Berenguera A, Almeda J, Violan C, Pujol E. Consideraciones sobre los estudios de prevalencia de fragilidad en el adulto mayor en España. Revista Atención primaria. 2012 44(5): 295-296.

7. Otero A, Castell M, Canto M. Cribado de fragilidad en atención primaria. Revista Española de Geriatría y Gerontología. 2011;46(5):239-240.
8. Shim EY, Ma SH, Hong SH, Lee YS, Paik WK, Seo DS, et al. Correlation between frailty level and adverse healthrelated: outcomes of community-Dwelling Elderly, one year retrospective study. Revista Korean J Fam Med. 2011;32(4):249-256.

9. Villagordoa J. Definición de envejecimiento y síndrome de fragilidad, características epidemiológicas del envejecimiento en México. Revista de endocrinología y nutrición. 2007; 15 (1): 27-31.

10. Varela-Pinedo L, Ortiz-Saavedra PJ, Chávez-Jimeno $\mathrm{H}$. Síndrome de fragilidad en adultos mayores de la comunidad de Lima Metropolitana. Revista Soc Peru Med Interna. 2008;21(1):12-15.

11. Abizanda P, Gómez J, Lesende I, Baztan J. Detección y prevención de la fragilidad: una nueva perspectiva de prevención de la dependencia en las personas mayores. Revista Medicina Clínica.2010;135(15):713-719.

12. Abizanda P, López-Torres J, Romero L, López M, Sánchez PM, Atienzar P, et al. Fragilidad y dependencia en Albacete (estudio FRADEA): razonamiento, diseño y metodología. Revista Española de Geriatría y Gerontología. 2011; 46(2):81-88.

13. Abizanda P. Actualización en fragilidad. Revista Española de Geriatría. 2010; 45(2):106-110.

14. Martínez C, Pérez V, Rosello A, Brito G, Gil M. La fragilidad: un reto a la atención geriátrica en la APS. Revista Cubana Med Gen Integr. 2005; 21(1-2) 1-7.

15. Romero AJ. Fragilidad: un síndrome geriátrico emergente. Revista electrónica de las Ciencias Médicas en Cienfuegos. 2010;8(6):81-90

16. Ramos D, Bettinelli LA, Pasqualotti A, Corso D, Brock F, Erdmann AL. Prevalencia de síndrome de fragilidad en los adultos mayores de una institución hospitalaria. Rev Latino-Am Enfermagem. 2013;21(4):[08 pantallas]

17. Quevedo-Tejero EC, Zavala-Gonzalez MA, AlonsoBenites JR. Síndrome de fragilidad en adultos mayores no institucionalizados en Emiliano Zapata, Tabasco, México. Univ Méd. 2011;52(3):255-268.

18. Gómez JF, Curcio CL, Henas GM. Fragilidad en ancianos colombianos. Rev Médica Sanitas. 2012; 15(4):8-16.

19. García F, Alfaro A. Fragilidad de la epidemiologia a la clínica. Revista Española de Geriatría y Gerontología. 2010;45(5):250-251.

20. García F, Larrion J, Rodríguez L. Fragilidad un fenotipo en revisión. Revista Gac Saint. 2011;25(S):51.58.

Para citar este artículo: Rivadeneyra-Espinoza L, Sánchez-Hernández C. Síndrome de fragilidad en el adulto mayor en una comunidad rural de Puebla, México. Duazary. 2016 julio; 13(2): 119 - 125 